\title{
La formación de competencias en la práctica virtual
}

\section{Skills training in virtual practice}

\author{
Karina Inés Schmidt \\ https://orcid.org/0000-0003-3310-4191 \\ karinaischmidt@gmail.com \\ Facultad de Odontología | \\ Universidad Nacional de La Plata | Argentina
}

\section{Agustina Morgante}

https://orcid.org/0000-0003-0947- 2939

agusmorgante@gmail.com

Facultad de Odontología |

Universidad Nacional de La Plata | Argentina

\section{Luis Alberto Salvatore}

https://orcid.org/0000-0003-1718-3520

betosalvatore@yahoo.com.ar

Facultad de Odontología |

Universidad Nacional de La Plata | Argentina

\author{
Viviana Carmen Serrano \\ https://orcid.org/0000-0001-5010-0841 \\ vivilulp@yahoo.com.ar \\ Facultad de Odontología | \\ Universidad Nacional de La Plata | Argentina
}

Miguel Pedro Mogollón

Carmelino

https://orcid.org/0000-0002-3803-0622

miguelmogollon47@gmail.com

Facultad de Odontología |

Universidad Nacional de La Plata | Argentina

Andrea Tanevitch

https://orcid.org/0000-0002-7797-7659

atanevitch@yahoo.com.ar

Facultad de Odontología

Universidad Nacional de La Plata | Argentina

\section{RESUMEN}

En la Tecnicatura de Asistencia Odontológica, desde el mes de marzo en que se declaró el Aislamiento Preventivo Social y Obligatorio (APSO) y hasta la actualidad, implementamos actividades virtuales sincrónicas y asincrónicas para el desarrollo de los contenidos temáticos del programa. Para crear un contexto de enseñanza y aprendizaje, utilizamos el entorno virtual de la plataforma institucional y videoconferencias que además de presentar baja complejidad en el manejo, accesibilidad desde distintos dispositivos, posibilitaron la interacción dinámica de manera colaborativa. El desafío consistió en elaborar una propuesta que permitiera desarrollar los conocimientos, habilidades, actitudes y valores relacionados con la asistencia en la atención odontológi$\mathrm{ca}$, de acuerdo a las normativas vigentes. Esta asignatura contempla evaluaciones de acreditación a través de las prácticas en servicio. No obstante, el seguimiento de los aprendizajes y la retroalimentación, resultan necesarios para la adquisición de las competencias pertinentes. Por ello, elaboramos una rúbrica para la evaluación de competencias de la práctica asistencial en el contexto virtual de enseñanza. La utilización de instrumentos cualitativos de evaluación contribuye a mejorar los procesos de adquisición de competencias y posibilitan el compromiso y autoevaluación del estudiante a la vez que promueven la cultura de la evaluación auténtica en la educación superior.

PALABRAS CLAVE competencias, TIC, evaluación, rúbrica. 
KEY WORDS

skills,

TIC,

assessment, rubric.

\section{SUMMARY}

At the Technician in Dental Assistance, since March when Obligatory Social Preventive Isolation was declared and until today, we have implemented synchronous and asynchronous virtual activities for the development of the thematic contents of the program. To create a teaching and learning context, we used the virtual environment of the institutional platform and videoconferences that, in addition to presenting low complexity in handling, accessibility from different devices, enabled dynamic interaction in a collaborative way. The challenge consisted in developing a proposal that would allow the development of knowledge, skills, attitudes and values related to dental care, in accordance with current regulations. This subject includes accreditation evaluations through practices made in service. However, the monitoring of learning and feedback are necessary for the acquisition of the relevant skills. For this reason, we elaborated a rubric for the evaluation of competences of assistance practice in the virtual teaching context. The use of qualitative assessment instruments contributes to improving the processes for the acquisition of competences and enables the student's commitment and self-assessment while promoting the culture of authentic assessment in higher education. 


\section{INTRODUCCIÓN}

Las innovaciones podemos entenderlas como cambios planificados frente a demandas del momento para adaptarse a las necesidades de docentes y alumnos para continuar con el aprendizaje. Actualmente con la declaración de la pandemia de Covid-19, en el ámbito de la Facultad de Odontología (UNLP) se ha trasladado el aula física a la modalidad de educación a distancia mediada por tecnologías.

En la Tecnicatura de Asistencia Odontológica, desde el mes de marzo en que se declaró el Aislamiento Preventivo Social y Obligatorio (APSO) y hasta la actualidad, implementamos actividades virtuales sincrónicas y asincrónicas para el desarrollo de los contenidos temáticos del programa. Utilizamos el entorno virtual de la plataforma institucional y videoconferencias para crear un contexto de enseñanza y aprendizaje que posibilite la interacción dinámica de manera colaborativa, mediante diversas herramientas informáticas que facilitan la gestión del conocimiento, la motivación, el interés, el autocontrol y la formación de sentimientos que contribuyen al desarrollo personal (Rodríguez, 2011). Con la utilización de las TIC se consigue interacción en tiempo real, además proveen un marco de convergencia tecnológica de gran cantidad de materiales de instrucción (videos, láminas de PowerPoint, imágenes planas, audio) y permiten aprendizajes sincrónicos con buen nivel de interacción para la formulación de preguntas y respuestas inmediatas (Rama, 2016).

A medida que el aislamiento dispuesto por las autoridades gubernamentales se fue extendiendo, nos encontramos con debilidades y obstáculos que emergieron reproduciendo las desigualdades sociales y culturales. Como sostiene Zabalza (2003-2004), los cambios deben ir acompañados de evaluaciones que permitan incorporar los ajustes necesarios para lograr efectividad y pertinencia.

Por ello decidimos realizar un relevamiento que dé cuenta de la experiencia de las estudiantes, en relación a la educación a distancia al finalizar el primer cuatrimestre. Las respuestas obtenidas de 13 alumnas de un total de 16 revelaron que los beneficios percibidos fueron en relación a la no concurrencia al establecimiento, la disminución de los gastos destinados al estudio, facilidad de acceso digital al material de estudio y el mejoramiento de su rendimiento académico. En cuanto a las dificultades, un $46 \%$ expresó tener cortes frecuentes de energía, un $15 \%$ no tenía computadora o no sabían manejar las herra- 
mientas virtuales, un 70\% manifestó dificultad para concentrarse en la clase sincrónica. Sólo un 30\% de las alumnas pudo entregar las tareas virtuales en el tiempo establecido e igual porcentaje consideró no haber tenido logros distintos en esta modalidad. Estos datos nos permitieron reflexionar acerca del diseño de la propuesta de enseñanza y la utilización de los recursos virtuales y la necesidad de contemplar la flexibilidad y accesibilidad de las cursantes. Como señala Davini (2008), es necesario reconocer la dimensión social de la enseñanza, en la cual participa el docente (o grupo de docentes) que actúa como un mediador entre los conocimientos y prácticas culturales y las personas que aprenden con características, necesidades y el contexto sociocultural específico. Pero además, una propuesta orientada a reforzar la adquisición de competencias profesionales de la práctica de asistencia odontológica, de acuerdo a las condiciones de la educación a distancia.

\begin{abstract}
Las respuestas obtenidas de 13 alumnas de un total de 16 revelaron que los beneficios percibidos fueron en relación a la no concurrencia al establecimiento, la disminución de los gastos destinados al estudio, facilidad de acceso digital al material de estudio y el mejoramiento de su rendimiento académico. En cuanto a las dificultades, un $46 \%$ expresó tener cortes frecuentes de energía, un $15 \%$ no tenía computadora o no sabían manejar las herramientas virtuales, un $70 \%$ manifestó dificultad para concentrarse en la clase sincrónica.
\end{abstract}

\title{
DESCRIPCIÓN DE LA EXPERIENCIA
}

La asignatura Prácticas Pre-Profesionales Supervisadas pertenece al segundo cuatrimestre del último año de la carrera de la Tecnicatura en Asistencia Odontológica es una materia totalmente práctica donde las/los asistentes realizan su formación final pre-profesional en los servicios de atención a pacientes, cuyos operadores pueden ser estudiantes del último año de la carrera de odontología o profesionales graduados. Este año nos vimos atravesados, como todo el sistema educativo, por la pandemia que nos convocó a reinventar la práctica de enseñanza y aprendizaje para adaptarla a la virtualidad.

En la modalidad presencial, las prácticas se realizan en el Hospital Odontológico Universitario o en el servicio de Prácticas Profesionales Supervisadas de la carrera de grado. La finalidad es desarrollar y consolidar competencias inherentes al perfil profesional del asistente odontológico. 
En la modalidad presencial, las prácticas se realizan en el Hospital Odontológico Universitario o en el servicio de Prácticas Profesionales Supervisadas de la carrera de grado. La finalidad es desarrollar y consolidar competencias inherentes al perfil profesional del asistente odontológico.

Tejada (2005) define a las competencias como: "todo un conjunto de conocimientos, procedimientos y actitudes combinados, coordinados e integrados, en el sentido que el individuo ha de saber hacer y saber estar para el ejercicio profesional. El dominio de estos saberes le hacen capaz de actuar con eficacia en situaciones profesionales" (p.7).

Se debe diferenciar la competencia de la capacidad. "La competencia no reside en los recursos (capacidades), sino en la movilización misma de los recursos. No son reducibles ni al saber, ni al saber hacer puesto que no se basan en saber imitar o aplicar rutinariamente los saberes del individuo sino saber actuar, que a veces implica no actuar" (Tejada, 2005, p.8). En la formación de profesionales, tradicionalmente la experiencia representaba la mayor capacidad de resolver problemas con un mayor nivel de cualificación. Sin embargo, en la actual sociedad del conocimiento, la experiencia y las cualificaciones están sujetas al vertiginoso ritmo del cambio. Esto exige que, para desarrollar competencias, lo adecuado es articular formación y experiencia, no sustituyendo una por otra. De allí la necesidad de planificar el espacio del prácticum pues la competencia se define en la acción (Tejada, 2005).

En el contexto del ASPO, las prácticas en servicio fueron suspendidas y se implementaron clases virtuales para asegurar la continuidad pedagógica. El desafío consistió en elaborar una propuesta que permitiera desarrollar conocimiento, habilidades, actitudes y valores relacionados con la asistencia en la atención odontológica.

En el contexto del ASPO, las prácticas en servicio fueron suspendidas y se implementaron clases virtuales para asegurar la continuidad pedagógica. El desafío consistió en elaborar una propuesta que permitiera desarrollar conocimiento, habilidades, actitudes y valores relacionados con la asistencia en la atención odontológica.

Como sostiene Ambròs (2009), "al momento de programar la unidad didáctica por competencias es importante que el alumnado 
perciba la funcionalidad y utilidad de la unidad de programación" (p.27). Por eso resulta relevante proponer tareas que tengan un sentido práctico de utilidad. En este aspecto, la unidad didáctica se organiza alrededor de un hilo conductor que resulte significativo para la práctica. Los objetivos por competencias implican tres tipos de saberes: el saber hacer, el saber y el saber estar y ser. Dichos saberes se corresponden perfectamente con conceptos, procedimientos, actitudes, valores y normas (Ambròs, 2009).

Esta asignatura comprende varios días de la semana y en cada uno hay un equipo docente a cargo. Se trabajó con un eje estructurador de la propuesta mediante la atención de pacientes virtuales a partir de los cuales cada equipo docente abordó un aspecto concerniente a la práctica clínica. En nuestro caso, los contenidos se organizaron en cuatro bloques temáticos.

- Unidad 1. Organización y administración del consultorio odontológico

- Unidad 2. Sistemas de comunicación eficiente

- Unidad 3. Documentación odontológica

- Unidad 4. Bioseguridad

Esta organización constituye un marco articulado y flexible mediante el cual se recuperaron conocimientos ya enseñados pero desde la coyuntura actual del contexto social y profesional de la epidemia. Como sostiene Maggio (2014) en relación a los programas "las articulaciones constituyen modos retrospectivos de mirar lo ya enseñado en otro nivel de análisis y a partir de dimensiones diferentes que permiten revisar su significado" (p. 67).

Para la implementación de la experiencia, utilizamos distintas estrategias y actividades que incluyen dramatizaciones, casos clínicos simulados, discusión y debate de situaciones problematizadoras. Davini (2008) considera dos modelos de enseñanza: la enseñanza entendida como instrucción y la enseñanza entendida como guía. En este sentido, la propuesta asumió el modelo de guía con un papel central de las actividades de quienes aprenden, la resolución de problemas, la reflexión activa y la inventiva. Se valoró la producción grupal de conocimiento y la actividad colaborativa.

De acuerdo a las concepciones didácticas manifestadas por Camilioni (2008), planteamos estrategias fundadas en el conflicto conceptual o cognitivo y en la teoría de la cognición situada. El conflicto cognitivo desde una dimensión social, permite la expresión pública de los modelos mentales que configuran el pensamiento de los estudiantes, la confrontación y la discusión de distintos puntos de vista y la validación y argumentación del docente para la construcción social del conocimiento. En la teoría de la cognición situada ad- 
quiere relevancia plantear situaciones de aprendizaje cuya actividad sea real y se corresponda con una práctica social. De esta manera se pretende reducir la brecha entre saber decir y poder emplear el conocimiento y generar aprendizajes que evidencian la interrelación entre actividad-cultura-concepto (Camilioni, 2008).

De acuerdo a las concepciones didácticas manifestadas por Camilioni (2008), planteamos estrategias fundadas en el conflicto conceptual o cognitivo y en la teoría de la cognición situada. El conflicto cognitivo desde una dimensión social, permite la expresión pública de los modelos mentales que configuran el pensamiento de los estudiantes, la confrontación y la discusión de distintos puntos de vista y la validación y argumentación del docente para la construcción social del conocimiento.

Se realizaron actividades sincrónicas y asincrónicas. En medicina y las ciencias de la salud el uso de videoconferencias sincrónicas respaldan la eficacia que posee la retroalimentación para los estudiantes, a la vez que posibilitan el desarrollo de contenidos, promueven habilidades procedimentales, sociales y de la comunicación para con los pacientes, docentes y sus pares (Reinoso-González, 2020). Los sistemas colaborativos sincrónicos, soportados en tecnología informática, son herramientas que favorecen la comunicación en tiempo real y crea un espacio dinámico de intercambio en el cual el estudiante puede recibir retroalimentación inmediata. Si bien existen distintas plataformas colaborativas disponibles, se utilizó Zoom puesto que presenta algunas ventajas como facilidad de uso, conexión sencilla e interactividad, operatividad desde distintos dispositivos. La principal desventaja de Zoom es la duración de 40 minutos de uso libre.

A continuación mostramos una actividad en torno a la paciente virtual Juana, que debe ser asistida contemplando las normativas y recomendaciones vigentes para la atención odontológica. El caso clínico se planteaba en el encuentro sincrónico abordando distintos aspectos referidos a la actuación de la asistente (Cuadro 1). Además se proponían actividades asincrónicas mediante el recurso: Foro de la plataforma Moodle. Allí las participantes hacían su aporte leyendo lo que sus compañeras habían escrito. Este recurso permitió además flexibilizar la participación en los casos que algún inconveniente haya dificultado la presencia sincrónica. Otras actividades consistieron en prácticas de ejecución como se muestran en el Cuadro 2. 
Consigna 1. CASO CLÍNICO 1- JUANA BASTARRICA

Juana tiene un problema odontológico y llama al consultorio dental para solicitar un turno. Ustedes son recepcionistas telefónicas y atienden el llamado. ¿Qué preguntas deberían realizar por teléfono a Juana antes de que concurra a la cita?

\section{Consigna 2.}

En esta actividad Juana llega al consultorio para la atención. ¿Qué medidas de protección y barreras de bioseguridad debe tener el paciente, el/la profesional actuante y la asistente? Considerar desde su llegada a la consulta hasta el momento que se le va a realizar la práctica.

\section{Consigna 3.}

En este caso Juana llega al consultorio para la atención. Cuando se presentó a la consulta el odontólogo se había retirado. ¿Qué falla ocurrió entre los integrantes del equipo de trabajo? ¿Se cumplió el protocolo de Covid-19 en la recepción del paciente? ¿Pueden detectar fallas? ¿Qué posibles soluciones puedes plantear para resolver el problema?

\section{Consigna 4.}

En esta actividad Juana está siendo atendida en el consultorio y no hay insumos para completar la práctica profesional. ¿Cómo podrían resolver la situación? ¿Qué alternativas puedes proponerle al profesional? ¿Cómo se las plantearías? ¿Cómo se podría haber evitado el problema? ¿Qué competencias tendría que tener la asistente para evitar la falta de insumos? ¿Cómo organizarías los diferentes insumos para la atención? ¿Cómo debería estar organizado un consultorio al momento de la atención del paciente? ¿Qué maniobras corresponde realizar para acondicionar el consultorio después de la atención?

\section{Cuadro 2. Actividades prácticas}

\section{Consigna 5.}

La actividad consiste en realizar un pedido de insumos a un proveedor de productos odontológicos. Para ello, de acuerdo a los productos publicados deberán confeccionar el pedido correspondiente. Para calcular los insumos necesarios deberán tener en cuenta las prácticas odontológicas realizadas en Juana y Francisco y la cantidad de sesiones que concurrieron a la atención. Recordar que las barreras de bioseguridad deben contemplarse para el paciente, el profesional y la asistente. Tener en cuenta que hay productos que deben adicionar el IVA al valor neto. El plazo de entrega es hasta el sábado 14 de noviembre subiendo el archivo en el Foro.

\section{Consigna 6.}

De acuerdo a las prácticas realizadas en Juana y Francisco, convertir los códigos del nomenclador unificado de Hospital Odontológico Universitario en los códigos del nomenclador de IOMA y realizar la facturación. Preparar la documentación necesaria para el reintegro

Esta asignatura contempla evaluaciones de acreditación a través de las prácticas en servicio. No obstante, el seguimiento de los aprendizajes y la retroalimentación, resultan necesarios para la adquisición de las 
competencias pertinentes. Anijovich (2010) expresa que la retroalimentación puede realizarse con foco en la autoestima o con foco en la tarea, siendo ésta la más productiva. Como sostiene la autora es importante considerar que "el estudiante no siempre va a producir una acción espontánea de revisión y/o mejora por el solo hecho de haber recibido una retroalimentación" (p.135). Para contribuir al cambio, es conveniente tener continuidad en el proceso de retroalimentación. Por ello, utilizamos una estrategia de construcción en espiral del conocimiento donde las actividades retoman aspectos críticos desde distintas dimensiones. El desafío consiste en generar una retroalimentación que le permita al estudiante detectar fortalezas y debilidades, obtener orientaciones y generar espacios sistemáticos para el intercambio de percepciones, experiencias y saberes que favorecerá el desarrollo de aprendizajes más profundos y desempeños más eficaces (Anijovich, 2010).

Para contribuir al cambio, es conveniente tener continuidad en el proceso de retroalimentación. Por ello, utilizamos una estrategia de construcción en espiral del conocimiento donde las actividades retoman aspectos críticos desde distintas dimensiones. El desafío consiste en generar una retroalimentación que le permita al estudiante detectar fortalezas y debilidades, obtener orientaciones y generar espacios sistemáticos para el intercambio de percepciones, experiencias y saberes que favorecerá el desarrollo de aprendizajes más profundos y desempeños más eficaces (Anijovich, 2010).

Valverde y Gómez (2014) sostienen que "actualmente se fomenta la evaluación orientada al aprendizaje, que se compone de los siguientes elementos básicos: participación activa del estudiante, pro-alimentación (retroalimentación prospectiva) y tareas auténticas". Este modelo de evaluación hace uso de estrategias que promueven y maximizan las oportunidades de aprendizaje de los estudiantes y es un "constructo teórico basado en la práctica universitaria". Tiene como finalidad fomentar el desarrollo de competencias útiles y valiosas para el presente académico y el futuro laboral (Valverde y Gomez, 2014).

La orientación de la formación universitaria centrada en el estudiante ha situado a la competencia en el eje central del diseño curricular y, por consiguiente, de la evaluación para el aprendizaje. Puesto que esta asignatura contempla evaluaciones de acreditación a través de las prácticas en servicio, el seguimiento de los aprendizajes y la retroalimentación, resultan necesarios para la adquisición de las competencias pertinentes. 
La rúbrica es un instrumento para la evaluación formativa que permite hacer explícitos los desempeños, los niveles de logro supuestos y los elementos subyacentes que deben ser explicitados y consensuados a la hora de emitir un juicio de valor. También puede ser una herramienta de autoevaluación, ya que orienta a los estudiantes a tener mayor control sobre su proceso de aprendizaje (metacognición).

\section{La rúbrica es un instrumento para la evaluación formativa que permite hacer explícitos los desempeños, los niveles de logro supuestos y los elementos subyacentes que deben ser expli- citados y consensuados a la hora de emitir un juicio de valor. También puede ser una herramienta de autoevaluación, ya que orienta a los estudiantes a tener mayor control sobre su proceso de aprendizaje (metacognición).}

Una rúbrica posee ciertos criterios o dimensiones a evaluar y lo hace siguiendo unos niveles o gradaciones de calidad y tipificando los estándares de desempeño. En cada casilla se describe que tipo de ejecución sería merecedora de ese grado en la escala (Alsina, 2013).

Así, construimos una rúbrica que se adaptara a los aprendizajes propuestos en el aula virtual y la presentamos a nuestras estudiantes al momento de plantearles las competencias que se espera adquieran y las tareas que llevan implícitas esas competencias. En el Cuadro 3 presentamos el detalle del instrumento elaborado.

Cuadro 3. Rúbrica para la evaluación formativa

\begin{tabular}{|l|l|l|l|l|}
\hline $\begin{array}{l}\text { COMPETEN- } \\
\text { CIA }\end{array}$ & \multicolumn{1}{|c|}{ CRITERIOS } & \multicolumn{2}{c|}{ NIVEL DE DESEMPEÑO } \\
\hline $\begin{array}{l}\text { Ser capaz de } \\
\text { registrar los } \\
\text { datos comple- } \\
\text { tos de la histo- } \\
\text { ria clínica con } \\
\text { autonomía. }\end{array}$ & $\begin{array}{l}\text { Completa todos los datos del } \\
\text { paciente. Realiza el odontogra- } \\
\text { ma al dictado. Realiza índices. } \\
\text { Registro y resguardo de la do- } \\
\text { cumentación legal. Codifica la } \\
\text { práctica odontológica realizada } \\
\text { para su facturación }\end{array}$ & $\begin{array}{l}\text { Cumple todos las } \\
\text { actividades en } \\
\text { forma autónoma }\end{array}$ & $\begin{array}{l}\text { Cumple } \\
\text { con las } \\
\text { actividades } \\
\text { con ayuda } \\
\text { parcial }\end{array}$ & $\begin{array}{l}\text { Cumple con las } \\
\text { actividades con }\end{array}$ \\
\hline $\begin{array}{l}\text { Desarrollar completa } \\
\text { habilidades } \\
\text { para la comu- } \\
\text { nicación con } \\
\text { el equipo de } \\
\text { salud y con la } \\
\text { triada pacien- } \\
\text { te-asistente- } \\
\text { profesional }\end{array}$ & $\begin{array}{l}\text { Utiliza lenguaje técnico con los } \\
\text { profesionales del equipo de } \\
\text { salud. Realiza interrogatorio de } \\
\text { salud al paciente asegurándo- } \\
\text { se la comprensión. Comunica } \\
\text { información al paciente para su } \\
\text { atención }\end{array}$ & $\begin{array}{l}\text { Cumple con todos } \\
\text { los criterios de } \\
\text { comunicación }\end{array}$ & $\begin{array}{l}\text { Cumple los } \\
\text { criterios } \\
\text { de comu- } \\
\text { nicación } \\
\text { con ayuda } \\
\text { parcial }\end{array}$ & $\begin{array}{l}\text { Cumple los crite- } \\
\text { rios de comu- } \\
\text { nicación con } \\
\text { ayuda completa }\end{array}$ \\
\hline
\end{tabular}




\begin{tabular}{|c|c|c|c|c|}
\hline \multirow[t]{2}{*}{ COMPETENCIA } & \multirow[t]{2}{*}{ CRITERIOS } & \multicolumn{3}{|c|}{ NIVEL DE DESEMPEÑO } \\
\hline & & DESTACABLE & ACEPTABLE & EN PROCESO \\
\hline $\begin{array}{l}\text { Desarrollar ha- } \\
\text { bilidades para la } \\
\text { comunicación con } \\
\text { el equipo de salud } \\
\text { y con la triada } \\
\text { paciente-asisten- } \\
\text { te- profesional }\end{array}$ & $\begin{array}{l}\text { Utiliza lenguaje técnico } \\
\text { con los profesionales del } \\
\text { equipo de salud } \\
\text { Realiza interrogatorio de } \\
\text { salud al paciente asegu- } \\
\text { rándose la comprensión. } \\
\text { Comunica información al } \\
\text { paciente para su atención }\end{array}$ & $\begin{array}{l}\text { Cumple con todos } \\
\text { los criterios de } \\
\text { comunicación }\end{array}$ & $\begin{array}{l}\text { Cumple los crite- } \\
\text { rios de comuni- } \\
\text { cación con ayuda } \\
\text { parcial }\end{array}$ & $\begin{array}{l}\text { Cumple los crite- } \\
\text { rios de comuni- } \\
\text { cación con ayuda } \\
\text { completa }\end{array}$ \\
\hline $\begin{array}{l}\text { Ser capaces de } \\
\text { administrar los } \\
\text { insumos del con- } \\
\text { sultorio }\end{array}$ & $\begin{array}{l}\text { Realiza inventarios y } \\
\text { calcula los insumos nece- } \\
\text { sarios para un periodo y } \\
\text { especialidad específicos } \\
\text { Muestra destreza para el } \\
\text { manejo de herramientas } \\
\text { informáticas }\end{array}$ & $\begin{array}{l}\text { Realiza las activi- } \\
\text { dades en forma } \\
\text { independiente } \\
\text { (autónoma) }\end{array}$ & $\begin{array}{l}\text { Identifica los in- } \\
\text { sumos necesarios } \\
\text { para las prácticas a } \\
\text { realizar pero nece- } \\
\text { sita ayuda para su } \\
\text { organización }\end{array}$ & $\begin{array}{l}\text { Necesita ayuda } \\
\text { para identificar y } \\
\text { organizar los in- } \\
\text { sumos necesarios } \\
\text { para la realización } \\
\text { de las prácticas a } \\
\text { realizar }\end{array}$ \\
\hline $\begin{array}{l}\text { Conocimiento } \\
\text { y manejo (colo- } \\
\text { cación y retiro } \\
\text { correcto) de los } \\
\text { EPP }\end{array}$ & $\begin{array}{l}\text { Describe el protocolo de } \\
\text { colocación, retiro y des- } \\
\text { carte del EEP }\end{array}$ & $\begin{array}{l}\text { Describe el proto- } \\
\text { colo de colocación, } \\
\text { retiro y descarte } \\
\text { del EEP en forma } \\
\text { completa }\end{array}$ & $\begin{array}{l}\text { Describe el } \\
\text { protocolo de } \\
\text { colocación, retiro } \\
\text { y descarte del EEP } \\
\text { con ayuda parcial }\end{array}$ & $\begin{array}{l}\text { Describe el } \\
\text { protocolo de } \\
\text { colocación, retiro } \\
\text { y descarte del } \\
\text { EEP con ayuda } \\
\text { completa }\end{array}$ \\
\hline
\end{tabular}

\section{CONCLUSIÓN}

En este trabajo mostramos nuestra experiencia, adaptaciones e innovaciones didácticas para favorecer el desarrollo de competencias mediada por el uso de tecnologías. Si bien la práctica profesional presencial es insustituible, el uso de las TIC nos brindó un abanico de posibilidades para proponer situaciones reales de aprendizaje favoreciendo la adquisición de competencias pre-profesionales adecuadas al escenario actual de pandemia.

La rúbrica nos permitió homogeneizar los criterios y hacer la evaluación más objetiva. Además, su elaboración contribuyó a descomponer la complejidad que implica el desempeño de la práctica clínica en criterios que describen acciones precisas que dan cuenta de distintos niveles de ejecución. En la asignatura pudimos implementar la rúbrica como instrumento de seguimiento y evaluación formativa, debido al reducido número de estudiantes pues su construcción insume tiempo si se detallan en forma precisa numerosas unidades temáticas.

La utilización de instrumentos cualitativos de evaluación contribuye a mejorar los procesos de adquisición de competencias y posibilitan el compromiso y autoevaluación del estudiante y promueven la cultura de la evaluación auténtica en la educación superior. 


\section{BIBLIOGRAFÍA}

Alsina Matmija J y otros (2013). “Rúbricas para la evaluación de competencias". Cuaderno de docencia universitaria 26. Ed. Octaedro. Barcelona, España.

Ambròs Alba (2009). “La programación de unidades didácticas por competencias". Aula de Innovación Educativa. 180, 26-32

Anijovich Rebeca (2010). “La evaluación significativa”. Buenos Aires: Paidós

Camilioni Alicia R. W de (2008). "El saber didáctico". Buenos Aires: Paidós

Davini María Cristina (2008). "Métodos de enseñanza: Didáctica general para maestros y profesores". Buenos Aires: Santillana

Maggio Mariana (2014).“Enriquecer la enseñanza superior: búsquedas, construcciones y proyecciones". InterCambios: Dilemas y Transiciones de la Educación Superior, 1 (1), 62-71

Rama C (2016). "La fase actual de expansión de la educación en línea o virtual en América Latina". Universidades (70), 27-39 Recuperado de https://www. redalyc.org/articulo.oa?id=37348529004

Reinoso-González Eduardo (2020). "La videoconferencia como herramienta de educación: ¿qué debemos considerar?". Rev. Esp. Edu. Med., 1:60-65 Recuperado de: https://revistas.um.es/edumed/article/view/426421/282881

Rodríguez M (2011). “Los entornos virtuales de aprendizaje como potenciadores del proceso educativo. Experiencia de su aplicación en la enseñanza presencial o semipresencial". Ponencia del XIV Congreso Internacional de Informática en la Educación, La Habana.

Tejada Fernández José (2005). “El trabajo por competencias en el prácticum: cómo organizarlo y cómo evaluarlo". REDIE, 7 (2), 1-31. Recuperado de: http://www. scielo.org.mx/scielo.php?script=sci_arttext\&pid=S1607-40412005000200013\&ln$\mathrm{g}=\mathrm{es} \& \mathrm{nrm}=\mathrm{iso}$

Valverde Berrocoso J y Gómez A (2014). "El uso de e-rúbricas para la evaluación de competencias en estudiantes universitarios". Estudio sobre fiabilidad del instrumento. REDU Revista de docencia Universitaria, 12. Universidad de Extremadura, España.

Zabalza Miguel (2003-2004). "Innovación en la Enseñanza Universitaria". Contextos Educ, 6-7, 113-136. 